\title{
La inclusión de nuevas tecnologías en las buenas prácticas de enseñanza de español en educación media*
}

\author{
Marina Gómez Azziz**
}

\section{Resumen}

Esta investigación trata de las buenas prácticas de enseñanza y el uso de nuevas tecnologías en las clases de Idioma Español, en educación media.

El marco teórico refleja una postura epistemológica relacionada con el paradigma posmoderno que reivindica el antipositivismo, el transaccionalismo y la valoración del contexto. Para ser coherentes con el enfoque anteriormente señalado, se eligió una metodología de investigación de corte cualitativo donde "no se buscan verdades últimas, sino relatos" (Valles, 1999, 56).

Creímos válido acercarnos al análisis de nuestro material de campo a través de tres dimensiones que consideramos globalizadoras. Ellas son: la dimensión espacio-temporal, la de abordaje y la de uso. Estas dimensiones tienen la mirada puesta en el docente y en las nuevas tecnologías, y nuestra mirada, en particular, se focalizó en el profesor y sus decisiones de aula al planificar su trabajo con nuevas tecnologías. A partir de ese primer enfoque se recorrió el quehacer del aprendiz, su actuar y su sentir respecto de las decisiones didácticas asumidas.

\section{Abstract}

This research focuses on good teaching practices and on the use of new technologies at high school Spanish lessons.

The theoretical frame reflects an epistemological viewpoint based on a postmodern paradigm that vindicates antipositivism, transactionalism and the value of context. A qualitative approach was selected in order to be coherent with the above stated perspective. "The search was not for ultimate truths, but for narrations." (Valles, 1999, 56).

We deemed convenient the analysis of our "field" material throughout three large globalising dimensions: place-time, approach and use. Said spheres focus on the teacher and the new technologies, with a much closer look on the teacher and their decisions within the classroom when planning the use of new technologies. From this first approach, the research went through the students'scene, their behaviour and their feelings towards the decisions taken.

\section{Introducción}

El tema de nuestra investigación es la inclusión de nuevas tecnologías en las buenas prácticas de enseñanza del español en educación media. El problema concreto, los modos en que se desplegó la enseñanza. El objetivo propuesto: analizar estas prácticas.

Este tema nos parece de vital importancia, ya que existe carencia de una positiva competencia en el uso que hacen de la lengua nuestros estudiantes, y sentimos la necesidad de reflexionar sobre nuestras acciones pedagógicas con trabajos que reflejen las miradas y concepciones de los distintos actores involucrados en el quehacer didáctico.

Cuando empleamos la expresión "nuevas tecnologías" (TIC), hacemos referencia a los medios tecnológicos de representación semiótica. A pesar de que algunos de ellos tengan muchos años en el mercado mundial en nuestro país, se han utilizado con poca frecuencia en los escenarios 
de actividad pedagógica a la hora de enseñar la lengua materna a nivel secundario, y no han sido motivo de investigación educativa en este campo. Nos referimos tanto a los grabadores y a las producciones cinematográficas como a Internet y a la telefonía móvil.

"No creo en últimas palabras en lo que a asuntos humanos se refiere, solo en mejores conversaciones". El ojo ilustrado, Eisner, E.,998, 21.

\section{Marco teórico}

El marco teórico refleja una postura epistemológica relacionada con el paradigma posmoderno que reivindica el antipositivismo, el transaccionalismo y la valoración del contexto. Para ser consecuentes con el enfoque anteriormente señalado, se eligió una metodología de investigación de corte cualitativo, en la que "no se buscan verdades últimas, sino relatos" (Valles $1999,56)$. En las ciencias humanas se vuelven a cuestionar la naturaleza de la mente y sus procesos, la construcción de nuestros significados y de nuestras realidades, y la formación de la mente por la historia y la cultura. Lo que conocemos no es absoluto, sino relativo a alguna perspectiva, a algún punto de vista.

Organizamos la presentación del marco teórico desde una temática abarcadora como es la tecnología, la cultura y el conocimiento; y desde esta perspectiva presentamos las diferentes posiciones teóricas respecto de la buena enseñanza y, especialmente, la buena enseñanza de la lengua, la tecnología y -como no podía ser de otra manera- el aprendizaje de la lengua hoy.

\section{Tecnología, cultura y conocimiento}

Al pensar en la tecnología, la cultura y el conocimiento, pensamos en dimensiones humanas estrechamente relacionadas entre sí. Si, como sostiene Bruner, la cultura conforma la mente y nos permite construir nuestros propios significados, ya no reconocemos una verdad única, sino diferentes apreciaciones de la realidad. Se ha reivindicado la visión individual, la subjetividad y también la intersubjetividad. Ya no hay absolutos en términos epistemológicos sino un reconocimiento de lo sincrónico y del contexto. A partir de los años ochenta comienzan a vislumbrarse cambios significativos en el campo de la didáctica. El paradigma posmoderno había iniciado su incursión en la filosofía y las ciencias. En concomitancia con dicho paradigma, se reconoció la necesidad de considerar la filosofía, la política, la ideología y la pedagogía como dimensiones ineludibles en el estudio de la enseñanza.

Se ha producido un cambio en la perspectiva del investigador. Las nuevas tecnologías, junto con otras que no lo son tanto, son consideradas soportes de la inteligencia repartida. La inteligencia es reconocida como parte de nuestro organismo, pero parte que tiene existencia más allá de él. Debemos hablar, pues, de una revolución en términos científicos. Ha habido un cambio radical en la concepción de lo que algunos autores llaman "mente". Si hablamos de inteligencia repartida, el soporte de información pasa a tener una relevancia hasta ahora no reconocida, y si, además, estos soportes nos permiten diferentes tipos de representaciones mentales, será insoslayable la utilización de aquellos a la hora de enseñar.

\section{Buena enseñanza}

Por buena enseñanza se entiende el abordar no solamente el núcleo duro de lo disciplinar, sino también asuntos morales que propicien en los alumnos el desarrollo de principios que mejoren sus propias vidas y la de su entorno. El docente debe saber mucho y estar al día para poder impartir buena enseñanza. No queremos dejar de señalar esto, que parece de Perogrullo. No 
obstante, el poseer un conocimiento profundo de determinado campo disciplinar no asegura que se puedan desplegar buenas prácticas. El profesor deberá ser capaz de seleccionar aquello que es adecuado para el alumno teniendo en cuenta su tiempo y su espacio vital; vale decir, es fundamental que se atienda a la plausibilidad de la información. Asimismo, al hablar de buena enseñanza no se puede dejar de mencionar la enseñanza comprensiva. Perkins (2001) y Litwin (2001) son los dos autores que mencionan y caracterizan este tipo de enseñanza como aquella que favorece procesos reflexivos en los alumnos y presenta analogías y contradicciones que se vuelven recurrentes en el análisis epistemológico. Pensar implica tener representaciones, afectos, motivaciones acerca de algo que de alguna manera conecta a la persona con el mundo. Pensar críticamente implica cuestionar, autocuestionarse y dialogar. Para poder potenciar estas actividades cognitivas, el propio docente debe construir sus comprensiones de esta forma.

\section{Buena enseñanza de la lengua española}

Los programas de la asignatura hace ya años que insisten en tres objetivos fundamentales. El primero de ellos es el siguiente:

"Lograr que el alumno se exprese oralmente y por escrito con corrección, eficacia y propiedad." (ANEP, 2006). Se explica en el documento señalado que correcto o incorrecto es cuestión de "aceptación social", según lo expresado por Amado Alonso. Se presenta, luego, una referencia al concepto de Coseriu de "diferencias diafásicas". El alumno debe ser consciente de la existencia de los diferentes registros y llegar a dominar el estándar.

El segundo objetivo señala que el discente debe lograr desarrollar y educar su capacidad para interpretar los contenidos lingüísticos. Respecto de este punto, vamos a señalar algunas reflexiones realizadas por Cassany en su libro "Tras las líneas -Sobre la lectura contemporánea (Montevideo, 2006). Digno de mención es el hecho de que el segundo capítulo comience con una cita de Foucault que reza: "El poder no está, por tanto, al margen del discurso. El poder no es ni fuente ni origen del discurso. El poder es algo que opera a través del discurso".

La dificultad radica en que los discursos no siempre son transparentes, como nos recuerda (Vattimo, 1998). Una de las formas más utilizadas de poder se da a través del discurso. Muchas veces, para descubrir el sentido amplio detrás de las líneas, debe poseerse una sólida cultura enciclopédica, por lo que también deberá fomentarse el conocimiento enciclopédico.Debemos propiciar la "literacidad" en los alumnos, en el entendido de que la "literacidad" es la capacidad de leer "las líneas, entre líneas y detrás de las líneas" (Cassany, 2006, 52). El autor hace referencia, especialmente, a poder recuperar los implícitos y construir el significado, a ser capaz de tener presente la connotación y no solamente la denotación.

Por último, se señala en el programa de la asignatura Idioma Español, como otro objetivo de la materia, procurar que el alumno adquiera un conocimiento reflexivo de la estructura de la lengua materna. La enseñanza de la lengua, como objeto de conocimiento desde un lugar especializado, se vincula a la explicitación de modelos o estructuras de pensamiento que se activan en la comprensión y producción de diferentes modalidades discursivas. Una modalidad discursiva se define, principalmente, por las funciones que cumple. La posición del interlocutor en la situación comunicativa supone una determinada actitud frente a lo que dirá, cómo lo dirá, a quién lo dirá y con qué objetivos. Se contemplan componentes pragmáticos, semánticos y sintácticos, además de lógico-racionales. Dichos componentes son fundamentales para el desarrollo sistemático de la competencia lingüística y comunicativa en situación de aula. 
La oralidad y la escritura cuentan con sistemas con leyes propias, independientes pero complementarios desde el punto de vista funcional.

La apropiación de la escritura permite desarrollar plenamente las potencialidades cognitivas y metacognitivas; no hay verdadera educación sin una completa apropiación de la lengua escrita.

El tema de la lectura mereció, dentro del programa, un apartado destacado. Transcribimos un enunciado:

"El adolescente actual se enfrenta a nuevas situaciones de lectura como las que realiza en el espacio cibernético-una interacción diferente a la estrategia de internalizar un texto impreso-porque la búsqueda de información se da mediante vínculos que determinan el manejo simultáneo de diversos textos" (ANEP, 2006). Teniendo presente la afirmación anterior, se le solicita al docente que lleve a cabo la orientación pedagógica del estudiante para que éste haga un uso crítico de la información en Internet dentro de la múltiple oferta del hipertexto.

Como autores de referencia para el tópico de la lectura digital debemos señalar a N. Burbules y a Th. Callister (2001) y su libro "Educación: riesgos y promesas de las nuevas tecnologías de la información". Burbules define el hipertexto como un formato que describe un entorno de información en el que el material textual y las ideas se entrelazan de diversas maneras. A medida que el procedimiento crece y evoluciona, la propia estructura de la información se modifica. El hipertexto se ocupa de asociar datos inconexos y también de cambiarlos mediante la identificación y creación de vínculos entre ellos. Supone conexiones figuradas, por lo que la forma y el contenido se vuelven interdependientes. Este hecho plantea cuestiones más profundas sobre el conocimiento.

"En el hipertexto, como en los textos en general, hay una relación interactiva entre su estructura y las estrategias de lectura que propone. Su forma, o las intenciones del autor al organizarlo de un modo particular, no determinan las maneras en las que puede ser recibido. Los comentarios marginales, notas y subrayados que agrega el lector suelen representar sus propias ideas acerca de las relaciones internas y órdenes de importancia, que a su vez incluyen enlaces con otras ideas a partir de las asociaciones establecidas durante la lectura" (Burbules y Callister, 2001, 80).

Al pensar en la buena enseñanza de la lengua española, debemos tener presente que se deberán propiciar escenarios de actividad para que el alumno se exprese oralmente y por escrito con corrección, eficacia y propiedad, para que logre interpretar los contenidos lingüísticos, y para promover el proceso reflexivo de la lengua. Para ello, en la actualidad no es suficiente con proporcionar al alumno textos orales y textos escritos de calidad. Hay que acercarlo, además, a la hiperlectura. Esta última, entendida como la decodificación del hipertexto, es coherente con un enfoque cognitivo que reconoce la mente y la memoria como hiperentornos.

Por último, pero no por ello menos importante, debemos tener presente que la información se transforma en conocimiento recién cuando la persona establece relaciones significativas. Por ello, el docente deberá favorecer los vínculos entre los diferentes temas y no dejarlos a la suerte de cada uno de los estudiantes.

\section{Tecnología y enseñanza}

Las nuevas tecnologías ya forman parte de nuestra cultura; no podemos desconocerlas. La sociedad se está tecnificando a pasos agigantados. Como docentes debemos habilitar oportunidades para que todos nuestros alumnos, sin excepciones, puedan acceder a estos medios. La socialización de las creaciones tecnológicas producirá un cambio cualitativo en 
nuestra sociedad. Las ideas que provocan -el efecto que queda luego de haber utilizado la tecnología- son de suma importancia. Esto es lo que Perkins, Salomon y Globerson Ilaman "residuo cognitivo" (Salomon, 1993), y se logra en contextos de atención deliberada y con control consciente. Lo más importante, según Buckingham (2008), no es el empleo de un solo medio, sino la convergencia de varios. La alfabetización en los medios se realiza mediante la interacción con ellos, y el papel que le cabe en este proceso a la institución educativa, es el de desarrollar el espíritu crítico y la capacidad creativa.

El hipertexto, caracterizado más arriba, impone una lectura interactiva y promueve formas de representación (Bruner, 2000) de distinta naturaleza, por lo que es útil para favorecer el aprendizaje. Jerome Bruner (2000) sostiene que se produce aprendizaje cuando se puede pasar de una forma de representación a otra, sea icónica, enactiva o simbólica, sin orden predeterminado.

Una de las limitaciones que tiene nuestra mente, explica Bruner (2000), está dada por los límites impuestos por el propio lenguaje. Y sin dejar de mencionar la hipótesis de Sapir y Whorf que afirma que el pensamiento toma su forma del lenguaje en el que se expresa, sostiene Bruner que la conciencia lingüística parece reducir las limitaciones de cualquier sistema simbólico. Por eso, lo metalingüístico, al decir de Jakobson, es algo que el profesor de lengua debe impulsar con todos los medios a su alcance. ¿Por qué no utilizar, también, las nuevas tecnologías como medios y que los docentes realicen las mediaciones necesarias para cultivar la conciencia metalingüística?

"El pensamiento sobre el pensamiento debe ser un ingrediente principal de cualquier práctica capacitadora de la educación" (Bruner, 2001, 38).

A propósito de mediaciones, es interesante el análisis que del término realizan tanto Burbules y Callister (2001) en el libro ya citado, como Jesús Martín-Barbero en "De los medios a las mediaciones" (1998). Según estos investigadores, todas las relaciones humanas son mediadas, incluso aquéllas que parecen muy directas, ya que en ellas interviene una variedad de gestos y rituales que afectan para bien o para mal esa comunicación. Un tipo de mediación es el canal o vía: la conversación cara a cara, la escritura, el teléfono u otros medios a distancia, entre ellos, Internet. Así, las interacciones sociales se vuelven diferentes según los medios a través de los cuales se articulan.

"Los diferentes tipos de comunicación, como los medios [...] fomentan modos de interacción que dejan traslucir y a la vez encubren; que encuadran lo que está y lo que no está abierto a la discusión; que fomentan determinados tipos de interacción (si se quiere determinados tipo de comunidad) y desalientan otros; $y$, consiguientemente, tienen efectos de inclusión y exclusión que afectan de manera diversa a diferentes participantes potenciales" (Burbules y Callister, 2001).

La comunicación se torna, muchas veces, en un asunto de "mediaciones" más que de medios; una cuestión cultural. El debate actual, según J. Martín-Barbero, está planteado en las articulaciones entre medios de comunicación y movimiento social, entre "prácticas comunicativas, socialidades y pluralidad de matrices culturales" (Martín-Barbero, 1998). La educación desempeña un papel sustancial. Uno de los fines de la Educación es la construcción del conocimiento hecha por todos, para que las personas sean más libres, más solidarias y más felices. A este respecto, y entre otros medios para lograr sus propósitos, el docente cuenta con las TIC. 
Todas las instituciones educativas, desde la escuela primaria hasta la universidad, están intentando establecerse como comunidades en y de Internet. Por lo tanto, resulta relevante estudiar lo político de la comunidad, porque limita o amplía las posibilidades.

"Espacio" y "lugar" son términos que se discuten y delimitan cuando se tratan la enseñanza y la tecnología. ¿Cuándo el primero se convierte en el segundo? El lugar se desarrolla cuando se percibe que se está en él; hay una sensación de familiaridad y reconocimiento. Tanto la familiaridad del espacio como la de las actividades lo moldean y respaldan. Las personas transformamos los espacios en lugares al actuar dentro y por encima de ellos para volverlos propios. A esta transformación se le llama "arquitectura". Así, pues, se habla de arquitectura en espacios públicos y privados, espacios textuales (por ej. la diagramación y el tamaño de un periódico), espacios agrícolas, educativos (los salones de clase, los pasillos, etc.), espacios digitales. Internet es, entonces, uno de ellos. Comprende numerosos medios de comunicación, los que operan sobre la base de la red de cables y ordenadores que constituyen la mencionada red (la World Wide Web o "telearaña mundial"): las páginas HTML, el correo electrónico, los "listservs" (sofware de gestión de lista de correo electrónico), grupos de discusión no simultáneos, IRC (Internet Relat Chat, protocolo de comunicación en tiempo real basado en texto), teleconferencias, videoconferencias, entre otros. Internet, en muchos casos, es un espacio al que la gente acude y con el que interactúa. En este sentido, se debe ser consciente de la diversidad, la tolerancia y la apertura de Internet.

Aquí, la labor de la educación es, como dijimos antes, enseñar a los lectores a ser individuos activos que reinterpreten y acomoden lo que encuentren en línea ("online") para transformarse así en hiperlectores, en lectores críticos.

Estas herramientas tecnológicas están instaladas en nuestra cultura y los docentes debemos aspirar a que todos nuestros educandos, sin excepción, alguna, accedan a ellas, porque siguiendo el pensamiento de Wolton, citado por Tedesco, el desafío no radica en tecnificar la sociedad, sino en socializar las técnicas (Tedesco, 2000).

Queremos insistir en que la nueva tecnología y la enseñanza en el siglo XXI, necesariamente deben ir de la mano. Son muchas las razones que así lo indican. Entre ellas destacaremos una que consideramos esencial: para tender a la equidad social, el hecho de que todos los alumnos tengan acceso a las nuevas tecnologías es fundamental. Aunque en otras latitudes este fenómeno ya se da por descontado, en nuestro país todavía hay un gran camino que recorrer para disminuir la brecha tecnológica que existe entre los alumnos con mayor poder adquisitivo y aquellos que provienen de contextos desfavorecidos.

\section{Aprendizaje de la lengua hoy}

Los datos estadísticos realizados por ANEP -MEMFOD (Administración Nacional de Educación Pública - Programa de Modernización de la Educación Media y Formación Docente) de los últimos años revelan aspectos duros de la realidad. Los datos obtenidos a través del Censo Nacional de Aprendizajes realizado en 1999, así como los resultados de OECD/PISA, 2003 en Idioma Español son realmente desalentadores, como muestran los cuadros realizados por la Dirección de Investigación, Evaluación y Estadística de la Administración Nacional de Educación Pública.

Colomer y Camps (1991) identifican cuatro niveles en la adquisición y el dominio de la lengua escrita: el nivel ejecutivo, el funcional, el instrumental y el epistémico. El nivel ejecutivo implica la capacidad de pasar un mensaje del modo escrito al hablado y viceversa. El segundo nivel es denominado funcional, ya que concibe la lengua escrita como un hecho de comunicación 
interpersonal que posibilita resolver las exigencias de la vida cotidiana. El nivel instrumental permite buscar y registrar información escrita; por ejemplo, cuando en matemática debe resolverse una tarea pero para ello es necesario interpretar un enunciado. En este caso, la lengua es un instrumento al servicio de la matemática. El estadio instrumental del lenguaje es el que permite el acceso a los demás conocimientos. El último nivel, el epistémico, implica el dominio del idioma y de una forma de pensar creativa y crítica. Teóricamente, en la educación secundaria, los dos niveles más trabajados deberían ser el tercero y el cuarto, pero dado que llegan los estudiantes con desconocimiento del nivel funcional y, desgraciadamente, cada vez más alumnos presentan carencias en el nivel ejecutivo, parecería que lo conveniente fuera el empleo de una articulación pedagógica de los cuatro niveles.

Cuando se habla de aprendizaje de la lengua o de aprendizaje en general, no se debe soslayar el papel crítico que juega la formación del yo. Aquí seguimos los caminos trazados por Bruner en su libro "La educación, puerta de la cultura" (2000). Hay dos aspectos del yo que se consideran esenciales. Uno es el denominado "agencia", y se reconoce por la sensación de poder iniciar y llevar a cabo actividades por cuenta propia. Las personas se sienten agentes.

"Lo que caracteriza a la persona humana es la construcción de un sistema conceptual que organiza un 'registro' de encuentros agenciales con el mundo, un registro que está relacionado con el pasado (es decir, la llamada memoria autobiográfica), pero que también está extrapolando hacia el futuro; un yo con historia y con posibilidad. Es un 'yo posible' que regula la aspiración, la confianza y el optimismo; y sus opuestos" (Bruner, 2000, 54).

La pedagogía moderna considera muy importante la conciencia del estudiante acerca de sus propios procesos de pensamiento. Por ello, es crucial ayudarlo a volverse cada vez más capaz de metacognición; es decir, ser tan consciente de cómo desarrolla su aprendizaje y su pensamiento, como debe serlo de los contenidos de la asignatura que está estudiando. No es suficiente con que sea hábil y posea conocimientos. Acercarlo a una buena teoría del aprendizaje ayuda al estudiante a adquirir un dominio general de su persona.

"La educación aporta los significados para reforzar y facilitar nuestras capacidades mentales innatas" (Bruner, 2000, 86).

Los contextos culturales que favorecen el desarrollo mental son interpersonales, ya que implican intercambios simbólicos. Cuando ocurre esta colaboración, el educando logra acceder a los recursos, a los sistemas simbólicos y, también, a la tecnología de la cultura. De acuerdo con las últimas investigaciones del aprendizaje humano, éste ocurre mejor cuando es participativo, proactivo, comunal, colaborativo y entregado a construir significados, más que a recibirlos.

"Aprender, en toda su complejidad, supone la creación y negociación en una cultura más amplia, y el profesor es el vicario de la cultura general" (Bruner, 2000, 102). Si esto es efectivo para cualquier aprendizaje, para el de la lengua es vital.

Un concepto central dentro de la teoría constructivista es el de que el docente no espera a que el estudiante esté psicológicamente preparado para recibir determinado saber; es él, como mediador, quien debe construir andamiajes para promover el desarrollo de las distintas capacidades. 
"Cualquier materia se puede enseñar a cualquier educando de cualquier edad, de una manera honesta" (Bruner, 2000,138).

El aprendizaje en general y el de la lengua en particular deben prestar atención a lo biológico y a lo cultural y ver cómo esos factores interactúan en situaciones locales.

Respecto al lenguaje, el investigador antes citado sostiene que algo en nuestro genoma nos permite acoger la estructura léxico-sintáctica de cualquier lengua natural y, asimismo, que es el lenguaje el que nos permite la construcción y elaboración de redes que constituyen los cimientos de la cultura.

Dada la temática de nuestra investigación, nos abocamos al sistema de la escritura y su impacto en la cultura y el individuo. Haciendo referencia a varios autores, expresamos que las primeras culturas urbanas, en el neolítico, en el delta del Tigris y el Éufrates, generaron nuevas formas de organización de la sociedad que necesitaron de un registro detallado que podríamos denominar, como lo señala Pozo $(2001,174)$, de "memoria externa". Así nace la escritura que, en su inicio, representaba el comercio agrícola y se realizaba en tablillas de cera. El empleo de la escritura trajo aparejados cambios en las representaciones mentales. La explicación a este fenómeno nos la proporciona Ong (1997). El habla oral y la escritura son dos dimensiones diferentes. La importancia de la escritura consiste no solamente en el hecho de que es un "ayudamemorias", sino también en el valor epistémico que conlleva. Nos permite pensar de otro modo, y el cambio implica un salto sustancial en la evolución de la humanidad, así como en el desarrollo de la persona en su ciclo vital. Según Olson (1996) es la escritura la que permite la metalingüística. No obstante, los efectos de la alfabetización y su análisis deben ser estudiados a partir de los modos de leer y no de los de la escritura. Si bien son procesos muy relacionados, son distintos. Leer diferentes tipos de textos (argumentativos, expositivos, descriptivos, entre otros) genera nuevas funciones mentales (Cassany, 2006).

Con la invención de la imprenta se extiende la relación entre el texto y la mente que producirá un nuevo tipo de conocimiento. Esto conduce a la aparición del tema de la interpretación. Interpretar los textos significa volver explícitas las posiciones epistémicas del autor y del lector. Los efectos cognitivos dependen, obviamente, de los usos sociales que de la escritura y la lectura se hagan. La "mente letrada", al decir de Olson (1996), es capaz de realizar representaciones del mundo (afirmaciones, ecuaciones, mapas, diagramas) y no trata el mundo en sí mismo. Esto significa hacer consciente el propio conocimiento. Este paso ha sido posible gracias a la escritura.

"Ni la ciencia ni el arte podrían entenderse sin los efectos de la escritura en la cultura"

(Pozo, 2001,178).

Nuestra comprensión del mundo, nuestra psicología es el producto de nuestras maneras de interpretar y crear textos escritos (Vygotsky, 1973). La función de la mente, por excelencia, es superar la entropía; es decir, debe ordenar sus representaciones de la realidad. Para hacerlo debe tomar conciencia de sí misma. Esta actividad se privilegia con la lectura y con una lectura crítica.

El término "tensión" fue acuñado por Meirieu (1992). La tensión implica curiosidad, "adrenalina", cierta presión psicológica que estimula. En el aula, la resolución de problemas puede producir tensión. Es necesario destacar que para que haya realmente aprendizaje, las informaciones deben estar circunscriptas a un proyecto, y entonces sí, se convertirán en conocimientos. 
¿Cómo podemos favorecer la superación de la entropía? Si explicamos a los estudiantes algunas teorías de la mente -como sugiere Bruner (2000)- con las cuales nosotros adherimos, y les explicamos el sistema de representaciones, sin que importen sus edades, seguramente facilitaremos el descenso del nivel de ansiedad, ampliaremos el umbral de frustración y desarrollaremos el deseo de aprender. Si a eso nosotros agregamos "tensión", la persona obtendrá logros positivos en su aprendizaje y vivirá las tareas educativas con agrado.

Meirieu (1992) sugiere crear un enigma, entusiasmar a los alumnos. Deben emplearse estrategias "accesibles y difíciles al mismo tiempo". Estas estrategias son tales cuando el alumno siente que es capaz de lograr el objetivo propuesto y percibe la existencia de una hipótesis que todavía no le es propia. Es importante recordar que estas estrategias deben ser variadas para favorecer los distintos estilos de aprendizaje. Así, pues, reconocemos las estrategias que emplean el apoyo verbal oral, las que utilizan el código escrito y las que hacen uso de un soporte visual. Otras son de corte auditivo; también las hay de contexto, cuando el individuo se apropia del conocimiento mediante la manipulación. Existen, asimismo, las estrategias de representación. Estas últimas utilizan el "signo" como mediador; emplean una imagen simbólica.

De lo expuesto inferimos que es conveniente crear momentos de reflexión en la clase, ya que "recrear el conocimiento y reconocerse supone cuestionar el autoritarismo [...] permite asumir la autoridad, desplegar la creatividad y la afectividad en los vínculos y evitar la repetición estereotipada y enajenante. Esto no implica la gratificación constante, sino al contrario, la sublimación del deseo del alumno en la búsqueda del conocimiento, en el ejercicio de su libertad, y en la posibilidad de asumir las frustraciones individuales e históricas que también son parte de su aprendizaje. Surgen entonces la autodisciplina y la constancia, pero también la integración de sí y de otros" (Fernando Gasalla, 2001, 115).

\section{Perspectiva metodológica}

Para recabar nuestro material empírico recurrimos a expertos en la comunidad académica, quienes nos condujeron a nuestros tres primeros docentes calificados. Solicitamos a estos tres docentes que nos informaran de otros profesionales que incorporaran TIC en sus clases y que fueran considerados profesores que impartieran buena enseñanza. Empleamos, así, el método de la bola de nieve. Nuestro universo de análisis quedó constituido por siete profesores de Montevideo (tres de instituciones privadas, dos de instituciones técnicas; tres de la periferia de nuestra ciudad, uno de institución bilingüe de clase media alta y alta).

El enfoque del trabajo fue diacrónico, en un estudio que duró aproximadamente un año. Por el tema seleccionado, para esta investigación consideramos relevante el empleo de las siguientes técnicas:

- observación de prácticas de aula;

- entrevistas semi-estructuradas a los docentes;

- análisis de los documentos que los docentes quisieran proporcionar;

- preguntas "flash" a algunos alumnos;

- triangulación.

El límite de nuestro universo de análisis estuvo señalado por la saturación. Tomamos el concepto de saturación del método comparativo constante de Glaser y Strauss, por lo que nuestro muestreo no estuvo dado por cálculos probabilísticos, sino por la saturación teórica. Es decir, reconocimos el límite de nuestro universo cuando el dato que se recolectó no aportó más información significativa a las categorías que se fueron construyendo. Solicitamos las autorizaciones correspondientes en todas las instituciones donde se trabajó. Las clases fueron grabadas, algunas filmadas, y se tomaron notas de campo, ya que todo elemento, actitud, expresión, entre otros índices, sería de utilidad a la hora de interpretar la realidad investigada. 
Creímos muy importante entrevistar a los docentes seleccionados para aproximarnos a las teorías de aprendizaje y de enseñanza a las que adherían o se sentían más próximos, y así acercarnos a sus representaciones, a su forma de ver el mundo. Asimismo, mantuvimos conversaciones informales con ellos, a posteriori de las experiencias de observación de clases.

Para estas entrevistas se los citó en un lugar "neutral". Por "neutral" entendemos un espacio que la persona no asocie inmediatamente al entorno estudiantil para que, en las referencias que haga en el coloquio, pueda apelar más a su interior. Una confitería nos pareció un ambiente apropiado para que los docentes estuvieran distendidos y más abiertos al diálogo. Los entrevistados fueron quienes seleccionaron la hora del encuentro, de modo que pudimos disponer del tiempo necesario para la aplicación de la técnica. Todas las entrevistas fueron grabadas, a excepción de dos. La no grabación de la primera de ellas se debió a un desperfecto técnico del dispositivo empleado. La segunda falla de grabación tuvo como causa que el docente a quien queríamos entrevistar se encontraba enfermo y no deseábamos comenzar las visitas de observación sin poseer la información que nos brindaría esa técnica. Por este motivo, el medio utilizado para la comunicación fue el correo electrónico. No obstante, es de destacar que este docente dispuso de tiempo extenso, después de las visitas que le realizamos a sus clases, para conversar con nosotros acerca de sus puntos de vista y sus interpretaciones de lo acontecido.

Trabajamos con entrevistas en profundidad, ya que son flexibles y dinámicas, y por ello permiten que el informante se exprese con mayor libertad. Así pues, formulamos una serie de preguntas que nos servirían de guía para la instancia planeada.

Se solicitó a todos los docentes algún material que quisieran compartir con nosotros. Obtuvimos tres planificaciones, algunas producciones con nuevas tecnologías realizadas por los alumnos, y trabajos estudiantiles de años anteriores, en los que se habían empleado estrategias de trabajo similares a las observadas por nosotros en esta oportunidad. Estos documentos, en algunos casos, nos hicieron comprender perspectivas y supuestos de quienes los habían producido, los que no se vieron tan claramente explicitados en otras técnicas.

Una vez que realizamos las entrevistas, comenzamos con las observaciones de clases. Estas clases debían presentar nuevas tecnologías. La técnica de observación implica un proceso contextualizado, al decir de Green (Wittrock, 1989), por lo tanto debimos prestar atención a factores que van más allá del aula. Por ejemplo, nos acercamos al conocimiento de la historia de la institución, ya que ésta supone una serie de expectativas, de tradiciones, de redes y de estilos de proceder que de alguna manera determinan el actuar en el aula. Se visitaron cuatro instituciones públicas; una de ellas se encuentra en el centro de la ciudad de Montevideo y las otras tres se ubican en barrios periféricos. Uno de esos barrios es considerado de contexto socio-económico crítico. Tres fueron las instituciones privadas visitadas. Una de ellas se encuentra en un barrio residencial de la capital de nuestro país y es centro de educación bilingüe. Ninguna de las tres instituciones privadas es confesional. Las dos que quedan por referir pertenecen a barrios de clase media, y ambas tienen un perfil tecnológico. Por ello, los programas correspondientes enfatizan especialmente en la tecnología.

Todas las clases fueron grabadas, y algunas de ellas también se filmaron. Asimismo se realizaron registros manuales donde describimos el espacio y los protagonistas, tomamos nota de la atmósfera de la clase, los gestos, y todos aquellos aspectos que no podían ser registrados por los dispositivos empleados. 
Nos pareció interesante y conveniente contar, también, con la mirada de los alumnos a propósito del empleo de las TIC en clases de Idioma Español para, así, acceder a contemplar "el triángulo pedagógico" (Meirieu, 1992).

Una vez finalizadas estas etapas, comenzamos a transcribir lo grabado y a leer los documentos que nos habían proporcionado los docentes. Iniciamos, así, nuestra tarea de análisis. Realizamos relecturas de las obras de nuestro marco teórico e incorporamos investigaciones publicadas durante la tarea de campo. Con el avance de la investigación comenzaron a surgir recurrencias y planos diferentes que nos fueron guiando para la construcción de nuestra perspectiva a propósito de las preguntas de investigación. En el corazón del trabajo se intentó entretejer las observaciones prácticas con la teoría, para proporcionar sentido a nuestra visión de la realidad en estudio. El período de validación de la investigación comenzó allí; es decir, buscando el sentido de lo observado a partir del conocimiento construido. Luego, creímos imprescindible contrastar todos los datos recogidos en la técnica de triangulación, para neutralizar las limitaciones que nos imponía el empleo de una técnica por separado.

\section{En busca y construcción...}

Nos acercarnos al análisis a través de tres dimensiones: la dimensión espacio-temporal, la de abordaje y la de uso. Estas dimensiones tienen la mirada puesta en el docente y las TIC. Nos enfocamos en el profesor y sus decisiones de aula cuando planificó trabajar con nuevas tecnologías. A partir de ese primer enfoque, abordamos el quehacer del aprendiz, su actuar y su sentir respecto de las decisiones didácticas que asumió el profesor.

\section{Dimensión espacio-temporal}

En esta dimensión se investiga en qué momento aparecen las nuevas tecnologías en el escenario didáctico y cuál es la intensidad con que se las emplea.

Se observan cuatro categorías: falla, presencia referida, categoría "estilo mariposa", y buceo. Estas categorías se construyeron teniendo en cuenta un "continuum" surgido del paralelismo que establecimos con la teoría de conjuntos de Georg Cantor (2005). El "continuum" es la representación geométrica del conjunto de los números reales. Nuestra representación de ese "continuum" estuvo dada por un segmento de recta, ya que acotamos nuestro análisis a aquellas prácticas de buena enseñanza en que habían sido empleadas las nuevas tecnologías.

De esta manera, en uno de los polos encontramos los escenarios de actividad donde las TIC lo acapararon todo, y en el otro, reconocimos una planificación de clase pensada para trabajar con nuevas tecnologías pero que la realidad impidió que se llevara a cabo. Por esto la denominamos "de la falla"1. En un caso el docente tuvo que recurrir necesariamente a otra planificación, y en la otra instancia observada, por desperfectos técnicos, tuvo que cambiarse de aula y poco faltó para que tuviera que alterarse, también, la planificación. La categoría buceo alude, metafóricamente, a un adentrarse en el mundo de las nuevas tecnologías como el buzo se sumerge al practicar su deporte. Y es desde esta inmersión como se realiza el proceso de enseñanza.

A mitad de ese "continuum" encontramos la categoría de la presencia referida y la del estilo mariposa. La presencia referida se encuentra más próxima al polo de la falla; no obstante, aquí no hubo elementos exógenos que interfirieran con las decisiones docentes; por el contrario, fue el docente quien decidió hacer uso de las nuevas tecnologías. En su discurso se valió de ellas, aludiendo a sus productos, pero éstos no fueron ejecutados dentro del aula. Es el caso del docente que emplea ejemplos de textos de "chats" y de mensajes de textos de teléfonos 
celulares para trabajar el tema de la oralidad y la escritura, pero los textos fueron producidos fuera del salón de clase. La categoría estilo mariposa se ubica cercana a la categoría buceo. Aquí el profesor no fomenta una total inmersión en el mundo de las TIC, pero hay un ir y venir permanente del mundo "virtual" al mundo "no virtual", y viceversa.

\section{Dimensión de abordaje}

Tiene que ver con las formas de aproximación que el docente hace mediante las TIC para lograr sus propósitos. Se observan dos categorías: la lingüística y la ícono-lingüística.

En la categoría lingüística el profesor comienza su andar didáctico a través del lenguaje. Por ejemplo, en una de las clases visitadas, analizamos situaciones de aula en donde los estudiantes eligieron, en la Red, avisos clasificados y escribieron textos que simulaban solicitudes de empleo. Otro docente propuso la co-evaluación, entre pares, de producciones escritas, empleando el ordenador.

La otra categoría presenta el uso, tanto lingüístico como icónico, en el abordaje de la escena pedagógica. La denominamos categoría ícono- lingüística. Observamos clases en las que, con el fin de un análisis generalizador y de comprensión, se introdujo al cine en el aula, donde los elementos semióticos extralingüísticos, los distintos planos de ficción y los "flashbacks" fueron analizados junto con lo lingüístico que presentó la película. Asimismo, se presenció una clase donde los alumnos debían crear una presentación en formato PowerPoint ${ }^{\circledR}$ para una muestra de fin de curso. En esas diapositivas los estudiantes debían seleccionar y presentar trabajos realizados durante el año lectivo que consideraran mejores o más próximos a ellos desde el punto de vista afectivo. Tenían que explicarlos verbalmente, empleando imágenes como apoyaturas.

\section{Dimensión de uso}

El foco de análisis corresponde al empleo que el profesor asigne a las nuevas tecnologías. Reconocimos dos categorías. A una de ellas la denominamos totalizadora, y a la otra distribuida. La primera señalada refiere a las situaciones en donde el docente concentra el poder de empleo de las TIC. Es el caso de una docente que creó un videoclip para emplear en su clase mientras se escuchaba y comentaba el contenido de una canción. La clase en cuestión tenía, como objetivo disciplinar, el acercamiento a la noción de la categoría gramatical de sustantivo. La docente misma creó el videoclip y dispuso del tiempo de aula para su presentación.

La categoría distribuida tiene más relación con el poder de uso de las TIC que tienen los alumnos. Mientras que la totalizadora presenta al profesor concentrando el poder de uso de las nuevas tecnologías, en esta categoría, por el contrario, el poder se ve distribuido entre los alumnos y son ellos quienes muestran más autonomía en el vínculo con las nuevas tecnologías. No obstante, es de destacar que en esta dimensión se está haciendo referencia concretamente a la relación de empleo de las TIC respecto del profesor y de los alumnos. El término "poder" está entendido solamente en este aspecto. Creemos que es oportuna esta aclaración ya que, en clases donde fue observada la categoría totalizadora, el clima de trabajo y la distribución de las tareas también correspondió al clima de buena enseñanza.

\section{Miradas sobre el estudiante}

Dado nuestro tema de investigación, nuestra atención estuvo focalizada primeramente en el accionar y en las decisiones de uno de los participantes en la relación pedagógica formal: el docente. Luego, centramos la atención en el otro actor, razón de ser de las decisiones pedagógicas adoptadas por el profesor: el alumno. Teniendo siempre presente que debemos 
diferenciar epistemológicamente el proceso de enseñanza del proceso de aprendizaje, no debemos descuidar que ambos están ontológicamente relacionados (Fenstermacher, en Wittrock I, 1989). En la mayoría de las clases observadas, los alumnos tuvieron la oportunidad de estudiar y producir textos correspondientes a distintos dominios de escritura. Tomando la taxonomía de Colomer y Camps (Cassany, 1998), podemos decir que se analizaron y se produjeron textos correspondientes al dominio ejecutivo, al funcional, al instrumental y al epistémico.

La motivación se vio potenciada con el empleo de las nuevas tecnologías. Esto se apreció en la disposición al trabajo y en las energías que invirtieron los alumnos en llevarlo a cabo. Algunos expresaron su interés cuando realizamos preguntas "flash" acerca del empleo de las nuevas tecnologías en las clases de Idioma Español. Otros alumnos se acercaron a nosotros y dieron su opinión. Percibimos como muy positiva la necesidad del alumno de compartir con nosotros su gusto por el espacio didáctico que acababa de vivir. Creemos que ese agrado que pudimos percibir puede haberse debido, entre otros aspectos, a la presencia de la "agencia", al decir de Bruner (2000). El docente permitió que el alumno realizara distintas tareas que le dieron confianza y optimismo. Se fomentó su autoestima.

Los alumnos tuvieron la posibilidad de ampliar su riqueza simbólica. Nuestro mundo nos brinda bienes materiales y bienes simbólicos, pero para valorar la riqueza simbólica es necesario contar con cierto nivel de cognición que permita procesar los diferentes sistemas de representación. Cuando este nivel de cognición fue reconocido como débil, el profesor instrumentó un andamiaje creado por él o por los integrantes de un grupo cooperativo. Como paradigmas de bienes simbólicos reconocemos el cine, la literatura, las artes plásticas, la música, entre otros. Estas diferentes manifestaciones del capital simbólico estuvieron presentes en la mayor parte de las clases.

Observamos que los alumnos debieron recrear el conocimiento en muchas propuestas de trabajo, por ejemplo, cuando tuvieron que producir textos de contenido similar al estudiado pero con diferentes superestructuras, o cuando se les solicitó la creación de diapositivas en formato PowerPoint ${ }^{\circledR}$. Estas situaciones les brindaron la oportunidad de "recrear el conocimiento y reconocerse, [...] y evitar la repetición estereotipada y enajenante" (Gasalla, 2001, 115).

\section{Conclusiones y consideraciones generales}

Dado que la perspectiva humana no es fotográfica sino interpretativa y " lo cotidiano es denso y debe ser mirado en detalle, porque en sus pliegues hay nudos de sentidos que no siempre quieren ser dichos" (Sarlo, 1996, 8) consideramos que no podía quedar silenciada la voz de los estudiantes, a pesar de que nuestro énfasis estuvo puesto en la perspectiva y el accionar docente. La actividad del docente cobra sentido en el vínculo con el alumno y construye su enseñanza a partir de él.

Por medio de trabajos de investigación crece el terreno teórico disciplinar. La investigación en el terreno de la educación y las nuevas tecnologías, no obstante, va detrás del rápido avance que llevan las innovaciones tecnológicas. A pesar de ello, somos conscientes de que el trabajo de análisis debe hacerse, porque da posibilidades al surgimiento de nuevas interrogantes y porque estimula la reflexión sobre el quehacer educativo.

En lo que respecta al marco teórico, tuvimos la oportunidad de observar desempeños que refieren a diferentes aspectos tratados por los investigadores. Muchos de los docentes hacen uso de las nuevas tecnologías porque éstas "están ahí y vinieron para quedarse". Forman parte del mundo cultural que nos toca vivir. Hay una visión ecológica de los materiales a los 
cuales recurrir. Estas TIC, muchas veces están en la institución, y por esta razón los docentes sienten que hay que adoptarlas y, en otros casos, adaptarlas, a las diferentes realidades de aula de las clases de español como lengua materna.

La mayor parte de los docentes reconoció el poder de motivación que estas tecnologías ejercen sobre los discentes. De las siete instituciones visitadas, dos son las que presentaron exigencias al docente en cuanto al uso efectivo de las TIC en el espacio pedagógico. Las otras cinco instituciones no se pronunciaron al respecto; no obstante, se observó una preocupación genuina por parte de los profesores por incorporar actividades de aula que requirieron el empleo de nuevas tecnologías. Dichos docentes son conscientes de la importancia que tiene en la vida de sus estudiantes el saber operar con estas nuevas herramientas.

Más allá de lo utilitario, demostraron ocuparse por despertar en sus alumnos una actitud de alerta a la hora de ver un producto cinematográfico, un videoclip, o una página de Internet. Fue reiterado el esfuerzo por inducir la reflexión y el análisis de cualquiera de estas realidades semióticas. Teniendo en cuenta las palabras de Cassany diremos que en todas las clases se vio un importante caudal de energía aplicado a lograr que el estudiante pudiera decodificar la línea, comprender entre líneas y llegar tras las líneas. La hiperlectura se reveló como uno de los objetivos reiterados. El uso de las TIC dependió, entre otros motivos, de lo disciplinar y del propósito de aula. La decisión del docente de emplear nuevas tecnologías porque consideró que la disciplina las requería hace presente la categoría de empleo genuino de las TIC (Maggio y Lion, en Litwin, 2005). Si bien hubo casos de docentes a quienes se les solicitaba un determinado porcentaje de clases en donde se emplearan nuevas tecnologías, fueron ellos quienes determinaron cuándo y cómo emplearlas, por lo que reconocimos la categoría genuina también en esos casos.

El uso de la lengua y la reflexión acerca de ella estuvieron presentes en todas las clases observadas. Podemos concluir diciendo que una buena planificación de clase donde se requiere un uso genuino de las nuevas tecnologías favorece la práctica lingüística y la metalingüística.

Otro de los aspectos que pudimos observar fue el trabajo colaborativo que permitió el empleo de las nuevas tecnologías. No obstante, tenemos presentes las palabras de Lion cuando expresa: "Las tecnologías pueden fortalecer el trabajo en colaboración, pero no crearlo" (Litwin, 2005, 201). Esta situación fue la que se reconoció cuando los alumnos tuvieron que utilizar la computadora. Aun en las aulas en que no tenían necesidad de compartir el ordenador, la colaboración fue una constante. Debemos señalar que el docente la propició a través de la palabra y de las actividades solicitadas. Hubo momentos de reflexión crítica acerca de las propias tareas y se apreciaron instancias de negociación para llegar a respuestas compartidas. Asimismo, todas estas experiencias se vivieron en un clima de absoluto respeto hacia el otro, lo que dio cuenta de un perfil de compromiso moral en los procesos de enseñanza, y también en los procesos de aprendizajes.

Después de realizar el trabajo, sentimos la necesidad de un abordaje de la temática desde lo institucional. Lo exige la etapa histórica que vive nuestro país, en la que se están implementando políticas educativas que fomentan la incorporación en las aulas de computadoras para todos los estudiantes y docentes. Será muy valioso disponer de aportes con investigaciones cuantitativas y cualitativas, donde las percepciones de los distintos actores involucrados en el quehacer educativo sean tenidas en cuenta y sus miradas sirvan para construir conocimiento en el campo de la didáctica y, particularmente, de la didáctica de la lengua española como lengua materna. 


\begin{tabular}{|c|c|c|c|c|c|c|c|}
\hline \multicolumn{8}{|c|}{\begin{tabular}{|l|} 
Administración Nacional de Educación Pública \\
Dirección de Investigación, Evaluación y Estadística
\end{tabular}} \\
\hline & & & & & & & \\
\hline \multicolumn{8}{|c|}{ Porcentaje de estudiantes de Educación Media por niveles de desempeño } \\
\hline \multicolumn{8}{|c|}{\begin{tabular}{|l|l|l|l|} 
en Lectura (2003) (a) (b). & & & \\
\end{tabular}} \\
\hline Niveles de & OCDE & Brasil & México & Uruguay & Argentina & Chile & Perú \\
\hline \multicolumn{8}{|l|}{ desempeño } \\
\hline 0 & 6.7 & 27.0 & 24.9 & 20.2 & 22.6 & 20.0 & 54.2 \\
\hline 1 & 12.4 & 23.1 & 27.1 & 19.6 & 21.3 & 28.2 & 25.4 \\
\hline 2 & 22.8 & 25.2 & 27.5 & 23.9 & 25.5 & 30.0 & 14.5 \\
\hline 3 & 28.7 & 16.5 & 15.6 & 19.8 & 20.3 & 16.6 & 4.9 \\
\hline 4 & 21.3 & 6.3 & 4.3 & 11.2 & 8.6 & 4.8 & 1.0 \\
\hline 5 & 8.3 & 1.9 & 0.5 & 5.3 & 1.7 & 0.5 & 0.1 \\
\hline Total & 100 & 100 & 100 & 100 & 100 & 100 & 100 \\
\hline
\end{tabular}

Fuente: Elaboración en base a datos de OECD/PISA 2003.

Nota (a): Los datos de la OCDE, Brasil, México y Uruguay son del año 2003. Los de Argentina, Chile y Perú, del 2001.

Nota (b): Los estudiantes que se encuentran en el nivel 1 o por debajo son aquellos que poseen una muy rudimentaria capacidad para leer, comprender y encontrar información en textos escritos de diverso tipo. Los estudiantes en los niveles 4 y 5 poseen capacidad para leer y comprender textos complejos, así como interpretar y reflexionar críticamente sobre el contenido de diversos tipo de textos.

\begin{tabular}{|c|c|c|c|c|}
\hline \multicolumn{5}{|c|}{\begin{tabular}{|l|} 
Administración Nacional de Educación Pública \\
Dirección de Investigación, Evaluación y Estadística
\end{tabular}} \\
\hline \multicolumn{5}{|c|}{$\begin{array}{l}\text { Porcentaje de suficiencia en Matemática e Idioma Español por forma de } \\
\text { administración según contexto sociocultural (a) en el Ciclo Básico de }\end{array}$} \\
\hline & Liceo público & Liceo privado & scuela Técnid & Total \\
\hline Matemática & & & & 56.3 \\
\hline Muy desfavorable & 39.1 & - & 29.9 & 35.5 \\
\hline Desfavorable & 47.8 & 63.7 & 35.2 & 46.7 \\
\hline Medio & 54.8 & 64.1 & -- & 55.3 \\
\hline Favorable & 60.2 & 81 & 33.3 & 68.8 \\
\hline Idioma Español & & & & 57.1 \\
\hline Muy desfavorable & 43.5 & -- & 31.2 & 38.5 \\
\hline Desfavorable & 49.6 & 64.2 & 31.8 & 48.1 \\
\hline Medio & 55.7 & 71.8 & -- & 56.6 \\
\hline Favorable & 58.3 & 82.3 & -- & 68.3 \\
\hline \multicolumn{5}{|c|}{ Fuente: Elaboración en base a datos del Primer Censo Nacional de Aprendizajes de } \\
\hline \multicolumn{5}{|c|}{$\begin{array}{l}\text { variable "promedio de años de educación materna" en cada centro. Para construir las } \\
\text { categorias de contexto se quintilizó esta variable y se elaboró un cruce por región. A } \\
\text { partir de esta estructura se imputaron los centros privados tomando los cortes de } \\
\text { años de educación materna elegidos. }\end{array}$} \\
\hline
\end{tabular}

\section{Bibliografía}

ANEP. 2006. Programas de Idioma Español - Ciclo Básico - Reformulación 2006. [Online]. [Citado 20 febrero 2008]. Disponible en Internet: <http://www.ces.edu.uy/menu_2006.htm>

Bruner, J. 2000. La educación, puerta de la cultura. Madrid. Aprendizaje Visor.

Buckingham, D. 2008. Más allá de la tecnología. Buenos Aires. Manantial.

Burbules, N.; Callister, Th. 2001. Educación: riesgos y promesas de las nuevas tecnologías de la información. Buenos Aires. Granica.

Cantor, G. 2005. Fundamentos para una teoría general de conjuntos: escritos y correspondencia selecta. Madrid. Crítica.

Cassany, D. 2006. Tras las líneas. Sobre la lectura contemporánea. Montevideo. Anagrama. 
Colomer, T.; Camps, A. 1991. Ensenyar a llegir, ensenyar a comprendre. Barcelona. Rosa Sensat. Ed. 62.

Cuban, L. 2002. Oversold and underused. Massachusetts. Harvard University Press.

Eisner, E. 1998. El ojo ilustrado. Indagación cualitativa y mejora de la práctica educativa. Barcelona. Paidós.

Gasalla, F. 2001. Psicología y cultura del sujeto que aprende. Buenos Aires. Aique.

Glaser, B.; Strauss, A. 1967. The Discovery of Grounded Theory. Strategies for qualitative research. New York. Aldine Publishing Company.

Irwin, J.; Doyle, M. (comp.). 1992. Conexiones entre lectura y escritura. Aprendiendo de la investigación. Buenos Aires. Aique.

Kuhn, T. S. 1991. La estructura de las revoluciones científicas. México. Fondo de Cultura Económica.

Liston D.; Zeichner, K. 1997. Formación del profesorado y condiciones sociales de la escolarización. Madrid. Morata.

Litwin, E. 2008. El oficio de educar. Buenos Aires. Paidós.

Litwin, E. (comp.). 2005. Tecnologías educativas en tiempos de Internet. Buenos Aires. Amorrortu.

Litwin, E. El campo de la didáctica. En: Camilloni, A., et al. 2001. Corrientes didácticas contemporáneas. Buenos Aires. Paidós.

Martín-Barbero, J. 1998. De los medios a las mediaciones. Bogotá. Convenio Andrés Bello. Meirieu, Ph. 1992. Aprender, sí. Pero ¿cómo? Madrid. Octaedro.

Olson, D.; Torrance, N. 1996. Cultura escrita y oralidad. Barcelona. Gedisa.

Ong, O. 1997. Oralidad y escritura. Tecnologías de la palabra. Buenos Aires. Fondo de Cultura Económica.

Perkins, D. 2001. La escuela inteligente. Barcelona. Gedisa.

Pozo, J. I. 2001. Humana mente - El mundo, la conciencia y la carne. Buenos Aires. Morata. Real Academia Española. 2001. Diccionario de la lengua española. Madrid. Espasa.

RAE y Asociación de Academias de la Lengua Española. 2005. Diccionario panhispánico de dudas. Bogotá. Santillana.

Salomon, G. 1993. Cogniciones distribuidas. Buenos Aires. Amorrortu.

Sarlo, B. 1996. Instantáneas. Medios, ciudad y costumbres en el fin de siglo. Buenos Aires. Ariel.

Tedesco, J. 2000. Educar en la sociedad del conocimiento. Buenos Aires. Fondo de Cultura Económica.

Valles, M. 1999. Técnicas cualitativas de investigación social. Reflexión metodológica y práctica profesional. Madrid. Síntesis.

Vattimo, G. 1998. La sociedad transparente. Barcelona. Paidós.

Vygotski, L. 2006. El desarrollo de los procesos psicológicos superiores. Barcelona. Crítica. Vygotski, L. 1973. Pensamiento y lenguaje. Buenos Aires. La Pléyade.

Wittrock, M. (comp.) 1989. La investigación de la enseñanza I, II, III. Barcelona. Paidós. 


\section{Nota}

${ }^{1}$ Cantor, (1845-1915), denomina conjunto vacío al que no posee elementos, pero que, a pesar de ello, continúa representando algo; representa la ausencia de elementos que pertenezcan a determinado contexto, el que está representado en el "continuum".

* El artículo se enmarca en la tesis de maestría de la autora, dirigida por la Dra. Lila Pinto. Año 2009.

** Máster en Educación, Universidad ORT Uruguay. Diploma en Educación, Universidad ORT Uruguay. Profesora de Educación Media - Especialidad Idioma Español, Instituto de Profesores Artigas. Profesora de Educación Media - Especialidad Inglés, Instituto de Profesores Artigas. 